\title{
Síntesis de Polioles por Modificación Química de Aceite de Algodón
}

\author{
Ilse C. Novoa D. ${ }^{a}$ y Erika Flores-Loyola ${ }^{a *}$ \\ ${ }^{a}$ Escuela de Ciencias Biológicas Universidad Autónoma de Coahuila. Carr. Torreón-Matamoros Km 7.5. Ciudad Universitaria s/n, \\ Torreón, Coah. \\ *E-mail:erika-flores@uadec.edu.mx
}

Recibido 16 de noviembre de 2012, Aceptado 14 de diciembre de 2012

\section{Resumen}

Los polioles son moléculas oligoméricas, comúnmente utilizadas para la síntesis de polímeros de gran interés industrial, que son obtenidas a partir de derivados del petróleo, por lo que es necesaria la búsqueda de nuevas materias primas renovables para su producción. El aceite de algodón, contiene un porcentaje alto de ácidos grasos insaturados, los cuales pueden ser transformados en polioles. En este trabajo se llevó a cabo la modificación química de aceite de algodón utilizando catalizadores heterogéneos y homogéneos. Se evaluó la influencia del medio (disolvente) y tiempo de reacción sobre el índice de hidroxilos y las propiedades químicas de los polioles obtenidos. Los resultados mostraron que la presencia o ausencia de disolvente no afecta de manera importante las características químicas de los polioles. Sin embargo el tiempo de reacción, así como el tipo de catalizador impacta directamente sobre el grado de modificación química del aceite de algodón lo que se observó en los índices de hidroxilo y los espectros de absorción FTIR de los respectivos polioles sintetizados.

Palabras clave: poliol, aceite de algodón, polimerización

\section{Introducción}

El petróleo es un recurso no renovable, es por ello que ha surgido interés en disminuir la dependencia de la industria de los polímeros con respecto al uso de los derivados de este producto. En este hecho recae la importancia de la búsqueda de nuevas materias primas renovables y ambientalmente benéficas. En los últimos años se ha estudiado el uso de aceites vegetales como fuente de materias primas, puesto que los aceites vegetales se componen de ácidos grasos poliinsaturados los cuales, mediante modificación química, pueden ser transformados a polioles moléculas oligoméricas, que pueden ser utilizadas como precursores de varios tipos de polímeros de interés comercial como los poliuretanos.

En la ruta de producción de polioles a partir de aceites vegetales, el aceite es sometido a una transesterificación, seguida de una epoxidación de los dobles enlaces de sus ácidos grasos insaturados y finalmente, el epóxido es hidrolizado para generar grupos hidroxilo en posición trans [1]. Por lo tanto, los aceites con un mayor grado de instauración dan lugar a polioles con mayor funcionalidad, lo cual es de suma importancia en los procesos de obtención de polímeros sintéticos [2]. Los aceites más estudiados en la producción de polioles han sido soya, canola, ricino, girasol y maíz por su alto grado de instauración. El aceite de algodón se compone de un $75 \%$ de ácidos grasos insaturados, el linoléico y oleico, lo que lo hace un excelente candidato para la obtención de polioles que pueden ser utilizados como precursores de polímeros diversos. A pesar de esto no existen, trabajos reportados en este aspecto, por lo que en el presente trabajo se plantea el uso de aceite de algodón como materia prima renovable en la obtención de polioles, evaluando las condiciones de reacción sobre las características químicas del producto.

\section{Parte Experimental}

Para la obtención de los polioles se utilizó aceite (Lirio S.A de C.V.), peróxido de hidrógeno (30\%, Faga lab), ácido acético glacial (99\%, Faga Lab), resina de intercambio iónico amberlita IRA400 (Supelco), tolueno (99\%, Faga Lab), $\mathrm{NaOH}$ (Fisher), acido sulfúrico al $99 \%$ (Faga Lab), $\mathrm{HBF}_{4}$ (48\%, Sigma), etanol (Analytica), $\mathrm{CaCl}_{2}$ anhidro (Baker). Para la determinación de índice de hidroxilos: $\mathrm{KOH}$ (Analytyka), 1-butanol (99 \%, Baker). La caracterización de los polioles se realizó mediante Espectroscopia IR en un equipo Nicolet IR100.

Para la obtención de polioles se realizaron 9 tratamientos, las condiciones de reacción de cada tratamiento se muestra en la tabla 1.

En los tratamientos 1 y 2 se llevó a cabo la epoxidación e hidrólisis ácida in situ, en donde 100 $\mathrm{g}$ de aceite algodón fueron puestos a reaccionar con $35 \mathrm{~g}$ de ácido acético glacial, en agitación y calentamiento en reflujo a $85^{\circ} \mathrm{C}$, posteriormente se agregaron lentamente $110 \mathrm{~g}$ de $\mathrm{H}_{2} \mathrm{O}_{2}$. Finalmente se adicionaron $0.2 \mathrm{~mL}$ de $\mathrm{H}_{2} \mathrm{SO}_{4}$ como catalizador, dejando reaccionar por $4 \mathrm{~h}$ a esa temperatura. 
Tabla 1. Condiciones de Reacción para obtención de polioles

\begin{tabular}{|c|c|c|c|c|c|c|c|}
\hline Tratamiento & $\begin{array}{c}\text { Tiempo de } \\
\text { reacción } \\
\text { horas } \\
\end{array}$ & ${ }^{\circ} \mathbf{C}$ & Catalizador & Disolvente & $\begin{array}{c}\text { Relación } \\
\text { molar } \mathrm{H}_{2} \mathbf{O}_{2}\end{array}$ & $\begin{array}{c}\text { Relación } \\
\text { molar ácido } \\
\text { acético } \\
\end{array}$ & $\begin{array}{l}\text { Reactivo de } \\
\text { apertura }\end{array}$ \\
\hline 1 & 4 & 80 & $\mathrm{H}_{2} \mathrm{SO}_{4} 1: 0.03$ & --------- & $1: 10$ & $1: 5$ & -------- \\
\hline 2 & 4 & 80 & $\mathrm{H}_{2} \mathrm{SO}_{4} 1: 0.21$ & --------- & $1: 10$ & $1: 5$ & -------- \\
\hline 3 & 24 & 60 & Resina An & Tolueno & $1: 5$ & $1: 2$ & $\mathrm{HBF}_{4}$ \\
\hline 4 & 16 & 60 & Resina An & Tolueno & $1: 5$ & $1: 2$ & $\mathrm{HBF}_{4}$ \\
\hline 5 & 8 & 60 & Resina An & Tolueno & $1: 5$ & $1: 2$ & $\mathrm{HBF}_{4}$ \\
\hline 6 & 24 & 60 & Resina An & --------- & $1: 5$ & $1: 2$ & $\mathrm{HBF}_{4}$ \\
\hline 7 & 16 & 60 & Resina An & ---------- & $1: 5$ & $1: 2$ & $\mathrm{HBF}_{4}$ \\
\hline 8 & 8 & 60 & Resina An & --------- & $1: 5$ & $1: 2$ & $\mathrm{HBF}_{4}$ \\
\hline 9 & 24 & 60 & Resina Cat & ---------- & $1: 5$ & $1: 2$ & $\mathrm{HBF}_{4}$ \\
\hline CONTROL 1 & 4 & 80 & --------- & --------- & $1: 5$ & $1: 5$ & -------- \\
\hline CONTROL 2 & 24 & 60 & --------- & --------- & $1: 5$ & $1: 2$ & $\mathrm{HBF}_{4}$ \\
\hline
\end{tabular}

Finalmente, se recuperó la fase orgánica del producto de reacción la cual se lavó con una solución saturada de $\mathrm{NaHCO}_{3}$, a continuación con una solución caliente de $\mathrm{NaCl}$ varias veces hasta $\mathrm{pH}$ neutro. Los remanentes de ácido fueron evaporados en una estufa a vacío a $60^{\circ} \mathrm{C}$ y los residuos de agua se eliminaron adicionando $\mathrm{CaCl}_{2}$ al producto obtenido. A modo de control se preparó la misma mezcla de reacción en ausencia de ácido sulfúrico.

En los tratamientos del 3 - 9 se realizó la epoxidación e hidrólisis por etapas, utilizando $100 \mathrm{~g}$ de aceite, $15.23 \mathrm{~g}$ de ácido acético glacial, $20 \mathrm{~g}$ de resina amberlita activada con ácido acético y $50 \mathrm{~mL}$ de tolueno según lo reportado por Guo et al. [2]. La mezcla fue agitada y calentada en reflujo a $60{ }^{\circ} \mathrm{C}$. Una vez a $60{ }^{\circ} \mathrm{C}$, se adicionaron $67 \mathrm{~g}$ de $\mathrm{H}_{2} \mathrm{O}_{2}$ lentamente, la reacción se mantuvo bajo estas condiciones durante 8, 16 y 24 h. Con el fin de evaluar el efecto del disolvente en el proceso de epoxidación se realizaron los mismos tratamientos en ausencia de tolueno [6]. La resina de intercambio iónico fue eliminada por filtración y la fase orgánica fue lavada varias veces con una solución caliente de bicarbonato de sodio, seguido de un lavado con agua caliente y una solución saturada de $\mathrm{NaCl}$ hasta $\mathrm{pH}$ 7. Posteriormente se agregó $\mathrm{CaCl}_{2}$ anhidro para eliminar los residuos de humedad. El tolueno remanente en el aceite epoxidado fue eliminado en una estufa a vacío a 60 ${ }^{\circ} \mathrm{C}$. A modo de control, se llevó a cabo la reacción anteriormente descrita en ausencia de resina de intercambio iónico, la cual funciona como catalizador del proceso de epoxidación del aceite de algodón $[1,5]$.
La reacción de formación del poliol (apertura del anillo oxirano) fue llevada a cabo colocando una mezcla de agua y metanol (30 g) en presencia de 1 $\mathrm{g}$ de $\mathrm{HBF}_{4}$ a la temperatura de ebullición de metanol, en reflujo, durante una hora con agitación continua. Posteriormente la mezcla fue enfriada a temperatura ambiente y se adicionó una resina de intercambio iónico IRA-400 activada con $\mathrm{NaOH}$ con el fin de eliminar los remanentes de ácido sin reaccionar. La resina fue eliminada por filtración y la fase orgánica resultante (poliol) fue lavada con agua hasta alcanzar un $\mathrm{pH}$ de 7 , posteriormente se adicionó $\mathrm{CaCl}_{2}$ para eliminar humedad [2]. Los polioles fueron caracterizados mediante espectroscopia IR utilizando el método de pastillaje con $\mathrm{KBr}$ anhidro. El número de hidroxilos presentes fue determinado mediante una titulación con solución alcohólica estándar de $\mathrm{KOH}$ siguiendo el método oficial 965.32 de la AOAC [4].

\section{Resultados y Discusión}

Se realizaron 9 tratamientos para llevar a cabo la modificación química del aceite, las condiciones de reacción de cada tratamiento se muestran en la Tabla 2. En la Tabla 5 se encuentran los resultados del índice de hidroxilos de los polioles obtenidos en cada uno de los tratamientos anteriormente descritos. Los resultados muestran que, de aquellos tratamientos en los que se utilizó resina como catalizador, solo los tratamientos 3, 6 y 9 dieron lugar a polioles, los cuales mostraron índices de hidroxilos con valores de 169.76, 197.76 y 157.68 $\mathrm{mg} \mathrm{KOH} / \mathrm{g}$ respectivamente, lo que indica la 
modificación química de los dobles enlaces característicos de los componentes insaturados del aceite de algodón por los grupos hidróxilos característicos de los polioles. El hecho de que los tratamientos 4, 5, 7 y 8 dieran resultados nulos de índice de hidróxilos indica que el tiempo necesario para que la reacción de hidroxilación pueda ser completada es de $24 \mathrm{~h}$ y que $16 \mathrm{~h}$ es insuficiente para este fin, estos tiempos son mayores a los reportados para la obtención de polioles a partir de otros aceites vegetales, sin embargo, no es posible hacer una comparación directa, ya que no existen reportes para la modificación química de aceite de algodón, no es posible determinar por qué varían los tiempos reportados por Petrović et al. [6]. Los polioles obtenidos en los tratamientos catalizados con resinas de intercambio iónico muestran índices de hidróxilos más altos que los reportados en la literatura. Narine et al. [7] reportan polioles de aceite de soya y canola por Ozonolisis con valores de índices de hidróxilos de 62.2 y $152.4 \mathrm{mg} \mathrm{KOH} / \mathrm{g}$ respectivamente. Por otra parte el poliol obtenido en el tratamiento 6 presenta un índice de hidróxilos (197.76mg KOH/g) comparable con los reportados por Petrović et al. [6] para aceite de soya hidroformilado, donde obtuvieron índices de hidróxilos de $160-230 \mathrm{mg} \mathrm{KOH/g}$.

El tratamiento 9 en que se utilizó una resina de intercambio catiónico durante 24 horas en ausencia de tolueno fue llevado a cabo con la finalidad de evaluar si la carga de la resina influía de manera importante sobre la eficiencia de la reacción. Los resultados mostraron que el poliol obtenido en este proceso presentó un índice de hidróxilos relativamente menor comparado con los polioles de los tratamientos 3 y 6 , lo que indica que la resina con carga negativa cataliza más eficientemente, en comparación con el ion hidróxilo $\mathrm{OH}^{-}$resultado de la reacción entre el ion $\mathrm{H}^{+}$de la resina de intercambio catiónico y la molécula de peróxido $\mathrm{H}_{2} \mathrm{O}_{2}$. Esto es debido a que, el ion de intercambio en la resina aniónica $\left(\mathrm{COO}^{-}\right)$actúa sobre la molécula de peróxido de hidrógeno $\mathrm{H}_{2} \mathrm{O}_{2}$ y la descompone para formar un ion perhidroxilo $\mathrm{OOH}^{-}$ [1], el cual reacciona rápidamente con una molécula de ácido acético $\mathrm{CH}_{3} \mathrm{COOH}$ para formar un peroxiácido, responsable de la epoxidación de los ácidos grasos insaturados del aceite de algodón. En los tratamientos 1 y 2 se utilizó acido sulfúrico como catalizador a diferentes concentraciones con el fin de evaluar el efecto de la concentración de este catalizador en la efectividad de la reacción (conversión de dobles enlaces a grupos hidróxilo).

Los resultados mostraron que el índice de hidróxilos de los polioles obtenidos fue independiente de la concentración de ácido sulfúrico utilizado. Esto es debido a que por ser el ácido sulfúrico utilizado como catalizador se requieren cantidades mínimas para llevar a cabo la hidrólisis ácida del aceite de algodón. Los polioles obtenidos por este método presentaron índices de hidróxilos de 284.57 y $284.9 \mathrm{mg} \mathrm{KOH} / \mathrm{g}$, los cuales son considerablemente mayores a los reportados por Petrović et al. [6] para la epoxidación de aceite de soya, el cual fue reportado con un índice de hidroxilos de $212 \mathrm{mg} \mathrm{KOH} / \mathrm{g}$, lo mismo sucede con los polioles reportados por Javni et al. [8] obtenidos por Ozonolisis de aceite de soya y canola con índices de hidróxilos de 260 y $228 \mathrm{mg} \mathrm{KOH} / \mathrm{g}$ respectivamente. Por lo tanto, los resultados obtenidos mostraron que la ruta de modificación por epoxidación resultó más eficiente que las rutas de Hidroformilación y Ozonolisis reportadas por los autores ya mencionados. Además los polioles obtenidos a partir de aceite de algodón obtuvieron índices de hidróxilos en algunos casos superiores a los polioles de aceites con porcentajes mayores de ácidos grasos insaturados como es el caso del aceite de soya y canola citados por la bibliografía [6 - 8].

En la tabla 2 puede observarse, que los procesos de obtención de polioles usando acido sulfúrico como catalizador fueron más eficientes que aquellos en que se utilizaron resinas de intercambio iónico. Esta efectividad fue establecida en función del mayor índice de hidróxilos obtenido para los polioles sintetizados en presencia de ácido sulfúrico. Así mismo, se debe considerar que el tiempo que tardan los tratamiento 1 y 2 para llevar a cabo la modificación química del aceite de algodón es mucho menor que los tratamientos en que se utilizan resinas ya que en estos casos es necesario realizar dos etapas independientes, una que considera una etapa de epoxidación y otra en la que el aceite epoxidado es hidrolizado en presencia de ácido fluorobórico. Sin embargo, en los tratamientos con ácido sulfúrico, las reacciones de epoxidación e hidrólisis del anillo oxirano se llevan a cabo en el mismo medio de reacción por lo que no fue necesario realizar el proceso en dos etapas. Esto es debido a que esta reacción, a diferencia de la llevada a cabo con resina, utiliza un ácido fuerte y agua, por lo que se genera, en el mismo medio de reacción, las condiciones requeridas para la apertura del anillo oxirano para generar los grupos $\mathrm{OH}$ característicos de los polioles. 
Tabla 2. Índice de hidroxilos de los polioles obtenidos por diferentes tratamientos

\begin{tabular}{ccc}
\hline Tratamiento & $\begin{array}{c}\text { Índice acidez } \\
\text { KOH } \mathbf{~ m g / g}\end{array}$ & $\begin{array}{c}\text { Índice de hidróxilos } \\
\text { KOH mg/g }\end{array}$ \\
\hline 1 & 3.9 & 284.57 \\
2 & 3.86 & 284.9 \\
3 & 1.17 & 169.76 \\
4 & 0 & 0 \\
5 & 0 & 0 \\
6 & 1.06 & 197.76 \\
7 & 0 & 0 \\
8 & 0 & 0 \\
9 & 1.07 & 157.68 \\
CONTROL 1 & 1 & 36.63 \\
CONTROL 2 & 0 & 0 \\
Aceite puro de & 0 & 0 \\
algodón & & \\
\hline
\end{tabular}

La efectividad como catalizador del ácido sulfúrico en comparación con las resinas de intercambio iónico es posiblemente debida a que las resinas pueden presentar problemas de difusión dado que como son catalizadores heterogéneos el $\mathrm{H}_{2} \mathrm{O}_{2}$ tiene que difundirse hacia el interior de los poros de la misma en los cuales se encuentran los iones disponibles para la reacción. Además, la formación del ion perhidroxilo $\mathrm{OOH}^{-}$producido por la resina de intercambio aniónico [9], reduce la eficiencia del $\mathrm{H}_{2} \mathrm{O}_{2}$, puesto que ocurre una reacción indeseable de descomposición de éste $(-\mathrm{OOH}+$ $\left.\mathrm{H}_{2} \mathrm{O}_{2} \longrightarrow \mathrm{O}_{2}+\mathrm{H}_{2} \mathrm{O}+-\mathrm{OH}\right)$ disminuyendo su capacidad de reaccionar con el ion $\mathrm{COO}^{-}$, reduciendo así la formación del ion perhidróxilo $\mathrm{OOH}^{-}$el cual reacciona con el ácido acético $\mathrm{CH}_{3} \mathrm{COOH}$ para obtener el peroxiácido.

En la Figura 1 se muestra el seguimiento, por espectroscopía IR, de los productos de las dos etapas de modificación química del aceite de algodón catalizada con resina a través de dos reacciones independientes: reacción de epoxidación y reacción de apertura de anillo (formación de poliol). Los resultados muestran, para el espectro del aceite de algodón puro (Figura 1a), las bandas correspondientes al aceite sin modificar. En 3005 y $1653 \mathrm{~cm}^{-1}$ se observan las bandas correspondientes a la vibración de tensión de los enlaces $\mathrm{C}-\mathrm{H}$ unido a doble enlace y $\mathrm{C}=\mathrm{C}$, respectivamente, presentes en los ácidos grasos insaturados característicos de este tipo de aceite [1], las cuales desaparecen tras la epoxidación (Figura 1b).

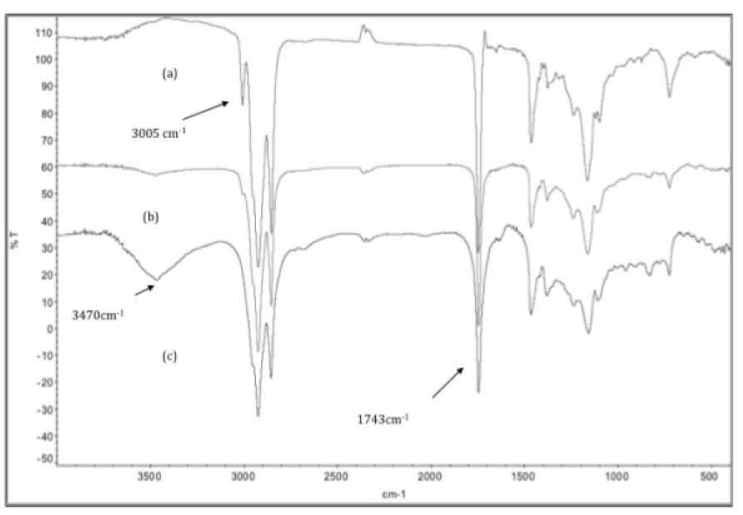

Figura 1. Espectros FTIR, Aceite puro de algodón (a), Aceite epoxidado (b) tratamiento 6 y Apertura del Epóxido (c) tratamiento 6.

Esto indica que la modificación química se llevó a cabo de manera efectiva. Posteriormente tras la reacción de apertura del anillo oxirano, aparece la banda en el $3470 \mathrm{~cm}^{-1}$ característica de la vibración de tensión de enlace O-H (Figura 1c), lo que indica que el grupo epóxido ha sido hidrolizado dando lugar a la formación del poliol que será utilizado como precursor de poliuretanos. Además, en los tres espectros aparecen una serie de bandas que se repiten, las cuales corresponden a las vibraciones de tensión del enlace $\mathrm{C}=\mathrm{O}$ en $1743 \mathrm{~cm}^{-1}$, características de los grupos carbonilo terminales de los ácidos grasos, y entre $2800-2900 \mathrm{~cm}^{-1}$ la vibración de tensión del enlace $\mathrm{C}-\mathrm{H}$.

En la Figura 2 se observa el seguimiento de la reacción catalizada con $\mathrm{H}_{2} \mathrm{SO}_{4}$. El espectro FTIR de la Figura 2a corresponde al aceite de algodón sin modificar y el $2 \mathrm{~b}$ al poliol obtenido después de someter al aceite a una modificación química catalizada con $\mathrm{H}_{2} \mathrm{SO}_{4}$ como se puede observar las bandas de tensión $\mathrm{C}=\mathrm{C}$ y $\mathrm{C}=\mathrm{C}-\mathrm{H}$ desaparecen después de que se lleva a cabo la reacción de formación de poliol, dando lugar a la aparición de la banda de tensión O-H características de los polioles. Esto indica que los dobles enlaces de los ácidos grasos insaturados del aceite de algodón fueron transformados directamente a grupos $\mathrm{OH}$ bajo las condiciones de reacción utilizadas en el tratamiento 1.

De los nueve tratamientos que se realizaron para la modificación química del aceite de algodón, solo en cinco de estos se obtuvieron polioles, los espectros de los polioles obtenidos en los cinco tratamientos efectivos se muestran en las Figuras 3, 4 y 5 . El espectro 3 b corresponde al control 1, en él no se empleó ningún tipo de catalizador. Como puede observarse, en este espectro se puede apreciar 
la presencia de una banda de muy baja intensidad en la región de $3470 \mathrm{~cm}^{-1}$ correspondiente a la vibración de tensión de enlace $\mathrm{O}-\mathrm{H}$ que indica que esta reacción pudo haber dado lugar a la formación de un poliol. Sin embargo, en este espectro se siguen observando la señal en $3005 \mathrm{~cm}^{-1}$ correspondientes a la tensión $\mathrm{C}=\mathrm{C}-\mathrm{H}$ lo que indica que en esta muestra existen aún los dobles enlaces de los ácidos grasos insaturados del aceite de algodón que permanecen sin modificar. Esto aunado al bajo índice de hidroxilos que reportó este tratamiento, es indicativo de una modificación en una proporción muy baja, la cual pudo ser debida a hidrólisis parcial de alguno de los componentes del aceite de algodón pero no necesariamente debida a la formación de polioles.

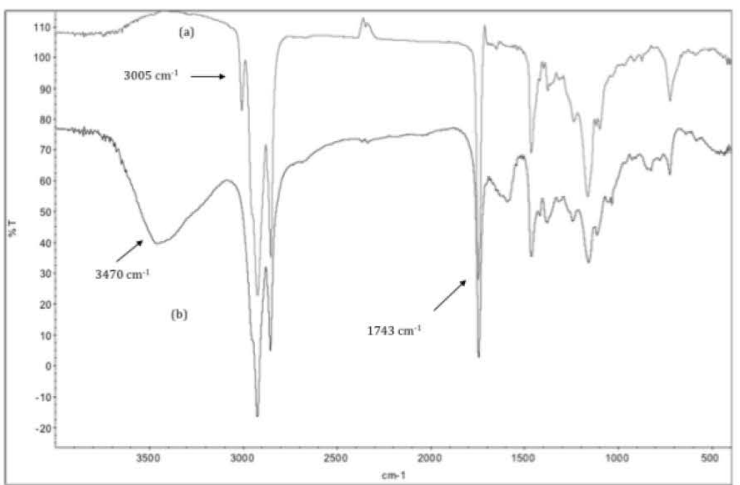

Figura 2. Espectro FTIR de tratamiento 1, Aceite de algodón (a) y Epoxidación-Apertura de Aceite de algodón (b).

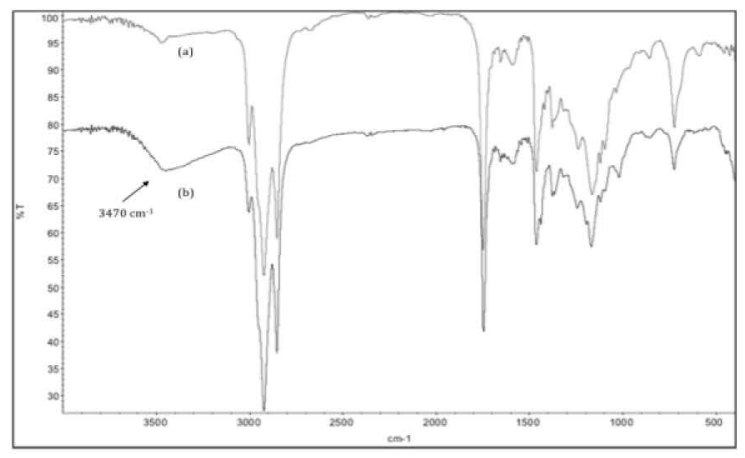

Figura 3. Espectros FTIR tratamientos sin catalizador, (a) Control 1, (b) Control 2 .

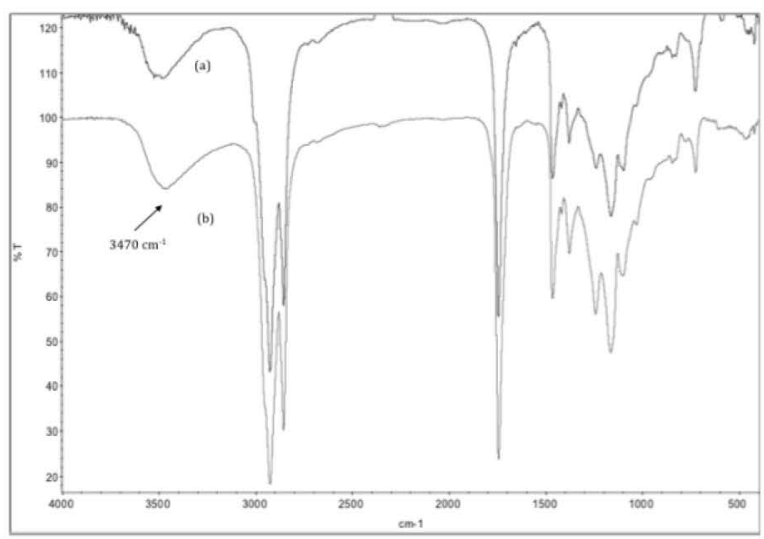

Figura 4. Espectros FTIR de tratamientos catalizados con resina, (a) tratamiento 3 con tolueno, (b) tratamiento 6 sin tolueno.

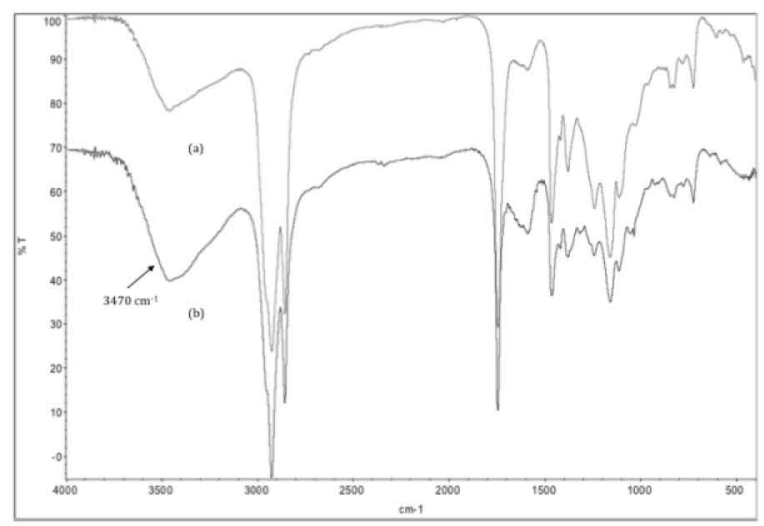

Figura 5. Espectros FTIR de los tratamientos catalizados con $\mathrm{H}_{2} \mathrm{SO}_{4}$, (a) tratamiento 2, (b) tratamiento 1.

El espectro de la Figura 3a corresponde al control 2 también libre de catalizador, en el cual no se observan cambios con respecto al espectro del Aceite sin modificar lo que indica que efectivamente es necesario la adición de un catalizador ácido ya sea en medio homogéneo o heterogéneo que permita que la reacción se lleve a cabo más rápidamente. De la Figura 4 puede apreciarse que la ausencia de tolueno como disolvente en la reacción no afecta la estructura química del poliol obtenido, lo cual se confirma también con el índice de hidroxilos para el poliol obtenido de esta manera, cuyo valor (Tabla 2) no presenta una diferencia significativa para ambos productos. Por otro lado, se observa (Figura 5) que la concentración de $\mathrm{H}_{2} \mathrm{SO}_{4}$ utilizado como catalizador tampoco afecta de manera importante la estructura química de los polioles obtenidos puesto que ambos espectros presentan las mismas bandas de absorción en el IR e índices de hidróxilos con valores muy cercanos entre sí. 


\section{Conclusiones}

Se obtuvieron polioles a partir de la modificación química del aceite de algodón (marca Lirio).

La presencia o ausencia de disolvente durante la reacción de obtención de polioles a partir de aceite de algodón, así como el uso de diferentes catalizadores, no afecta de manera significativa la estructura de los mismos. El tratamiento más eficiente para la obtención de polioles fue aquel en que se utilizó ácido sulfúrico como catalizador y cuyo tiempo de reacción es de $4 \mathrm{~h}$.

Los espectros FTIR de los polioles sintetizados en presencia o ausencia de tolueno fueron similares lo que indica es posible eliminar el tolueno como disolvente en la reacción de epoxidación del aceite de algodón sin que esto afecte la estructura química de los polioles obtenidos.

De los tratamientos 1 y 2 se obtuvieron polioles a partir de la modificación química del aceite de algodón con un índice de hidroxilos $(284.5 \mathrm{mg}$ $\mathrm{KOH} / \mathrm{gr}$ ) mayor a los reportados para polioles derivados de aceite de soya [5]. Además se logró establecer una técnica de obtención de polioles utilizando ácido sulfúrico como catalizador logrando que la hidrólisis del epóxido se llevará a cabo de manera simultánea a la formación del epóxido, obteniendo un tiempo de reacción total de $4 \mathrm{~h}$, el cual es mucho menor que los tiempos reportados por otros autores los cuales constan de 8 - $24 \mathrm{~h}$ para obtener un poliol, lo que indica que el aceite de algodón puede ser usado como materia prima para obtener polioles con un grado de funcionalidad comparable al de los obtenidos con los aceites comúnmente usados para este fin.

\section{Referencias}

1. Campanella, A.; Baltanas M. A. Catal. Today. 2005, 107, 208-214.

2. Guo, A.; Javni, I.; Petrović Z. J. Appl. Polym. Sci. 2000, 77, 467-473.

3. Espinoza, Perez J. D.; Haagenson, D. M.; Pryor, S. W.; Ulven, C. A.; Wiesenborn D. P. American Society of Agricultural and Biological Engineers 2009, 52(4), 12891297.

4. AOAC Official Method 965.32. Hydroxyl Value of Oils and Fats Acetylation Method. 1977.

5. Petrović Z.; Zlatanic, A.; Lava, C.; Sinadinovic-Fiser. Eur. J. Lipid Sci. Technol. 2002, 104, 293-299.

6. Petrović Z.; Wei, Zhang; Javni, I. J. Mater. Sci. 2006, 41, 4914-4920.

7. Narine, S.; Xiaohua, K.; Laziz, B. and Peter, S. Journal American Oil Chemical Society 2007, 84, 55-63.

8. Javni, I.; Petrović Z.; Wei, Zhang. Biomacromolecules 2005 , 6, 713-719.

9. Carretero, J. Epoxidación regioselectiva de diofelinas terpénicas mediandte catalizadores heterogéneos. Tesis Doctoral, Universidad Complutense De Madrid., Madrid, España. 2008. 